\title{
Variability in the Diagnosis and Treatment of Group A Streptococcal Pharyngitis by Primary Care Pediatricians
}

\author{
Julie L. Fierro, MD, MPH; ${ }^{1}$ Priya A. Prasad, MPH; ${ }^{1,2}$ A. Russell Localio, PhD; $;^{2,3,4}$ Robert W. Grundmeier, MD; ${ }^{5,6,7}$ \\ Richard C. Wasserman, MD, MPH; ${ }^{8}$ Theoklis E. Zaoutis, MD, MSCE; ${ }^{1,2,4,7}$ Jeffrey S. Gerber, MD, PhD ${ }^{1,2,4,7}$
}

\begin{abstract}
OBJECTIVE. To compare practice patterns regarding the diagnosis and management of streptococcal pharyngitis across pediatric primary care practices.
\end{abstract}

DESIGN. Retrospective cohort study.

SETTING. All encounters to 25 pediatric primary care practices sharing an electronic health record.

METHODS. Streptococcal pharyngitis was defined by an International Classification of Diseases, Ninth Revision code for acute pharyngitis, positive laboratory test, antibiotic prescription, and absence of an alternative bacterial infection. Logistic regression models standardizing for patient-level characteristics were used to compare diagnosis, testing, and broad-spectrum antibiotic treatment for children with pharyngitis across practices. Fixed-effects models and likelihood ratio tests were conducted to analyze within-practice variation.

RESULTS. Of 399,793 acute encounters in 1 calendar year, there were 52,658 diagnoses of acute pharyngitis, including 12,445 diagnoses of streptococcal pharyngitis. After excluding encounters by patients with chronic conditions and standardizing for age, sex, insurance type, and race, there was significant variability across and within practices in the diagnosis and testing for streptococcal pharyngitis. Excluding patients with antibiotic allergies or prior antibiotic use, off-guideline antibiotic prescribing for confirmed group A streptococcal pharyngitis ranged from $1 \%$ to $33 \%$ across practices $(P<.001)$. At the clinician level, 13 of 25 sites demonstrated significant within-practice variability in off-guideline antibiotic prescribing $(P \leq .05)$. Only 18 of the 222 clinicians in the network accounted for half of all off-guideline antibiotic prescribing.

CONCLUSIONS. Significant variability in the diagnosis and treatment of pharyngitis exists across and within pediatric practices, which cannot be explained by relevant clinical or demographic factors. Our data support clinician-targeted interventions to improve adherence to prescribing guidelines for this common condition.

Infect Control Hosp Epidemiol 2014;35(S3):S79-S85

The overuse of antibiotics has been associated with antibiotic resistance and adverse drug effects, which can result in increased healthcare costs and poor patient outcomes. ${ }^{1-3} \mathrm{Re}$ ducing inappropriate use of antibiotics is a critical step in slowing the progression of antibiotic resistance and improving quality of care. ${ }^{4}$ To date, antimicrobial stewardship efforts have been focused on inpatient care; however, the majority of antibiotic use occurs in outpatient settings. Identifying common outpatient infections for which antibiotics are often prescribed might provide opportunities for high-impact interventions.

Acute pharyngitis is an attractive target for antimicrobial stewardship because it is one of the most common indications for antibiotic prescribing to children and an objective, pointof-care laboratory test is recommended for the diagnosis of group A streptococcus (GAS) - the only etiology of pharyngitis warranting antibiotic therapy. ${ }^{5}$ GAS is the main bacterial cause of pharyngitis, accounting for 15\%-20\% of cases among children aged 5-15 years. ${ }^{6,7}$ The American Academy of Pediatrics (AAP), the Infectious Diseases Society of America (IDSA), and the Centers for Disease Control and Prevention $(C D C)$ have issued guidelines for the diagnosis and treatment of GAS pharyngitis. ${ }^{8-11}$ Furthermore, appropriate testing for children with pharyngitis is an outpatient quality measure in the Healthcare Effectiveness Data and Information Set, which is used to measure quality in managed care or-

Affiliations: 1. Division of Infectious Diseases, Children's Hospital of Philadelphia, Philadelphia, Pennsylvania; 2. Center for Pediatric Clinical Effectiveness, Children's Hospital of Philadelphia, Philadelphia, Pennsylvania; 3. PolicyLab, Children's Hospital of Philadelphia, Philadelphia, Pennsylvania; 4. Center for Clinical Epidemiology and Biostatistics, Perelman School of Medicine at the University of Pennsylvania, Philadelphia, Pennsylvania; 5. Division of General Pediatrics, Children's Hospital of Philadelphia, Philadelphia, Pennsylvania; 6. Center for Biomedical Informatics, Children's Hospital of Philadelphia, Philadelphia, Pennsylvania; 7. Department of Pediatrics, Perelman School of Medicine at the University of Pennsylvania, Philadelphia, Pennsylvania; 8. Department of Pediatrics, University of Vermont College of Medicine, Burlington, Vermont.

Received April 8, 2014; accepted April 13, 2014; electronically published September 15, 2014.

(C) 2014 by The Society for Healthcare Epidemiology of America. All rights reserved. 0899-823X/2014/35\$3-0012\$15.00. DOI: 10.1086/677820 
ganizations. Improving antibiotic use for GAS pharyngitis demands adherence to 2 basic principles: (1) judicious diagnosis, given that non-GAS pharyngitis-the majority of acute pharyngitis in children-does not require antibiotic therapy, and (2) narrow-spectrum therapy, which is uniformly recommended by national guidelines. Although antibiotic prescribing for pharyngitis has decreased, the rate of overall prescribing remains inappropriately high, and prescribing of broad-spectrum antibiotics continues to occur. ${ }^{12-15}$

Identifying variability in the diagnosis and management of GAS pharyngitis can provide opportunities for targeted interventions to reduce inappropriate antibiotic use. ${ }^{16}$ Prior studies have been limited by their inability to assess detailed clinical data, such as prior antibiotic use, antibiotic allergies, and comorbid medical conditions, and to compare trends across practices, which can provide useful benchmark data. ${ }^{6,13,17,18}$ Therefore, we aimed to compare rates of diagnosis, testing, and treatment for GAS pharyngitis in children by means of a large network of primary care pediatric practices linked by a common electronic health record (EHR).

\section{METHODS}

\section{Data Source}

Data were obtained from an ambulatory network of 222 clinicians from 25 primary care pediatric practice groups (ranging from 5 to 18 clinicians) located across southeastern Pennsylvania and southern New Jersey. A common EHR (EpicCare; Epic Systems), used exclusively by all practice sites for charting, laboratory testing, and medication prescribing for all office and telephone encounters since 2004, served as the data source.

\section{Data Collection}

Patient-level data extracted from the EHR included race (as reported by parent), sex, age in years, insurance type, and antibiotic allergies. Visit-level data included practice site, calendar month of contact, encounter type (office visit, telephone call, emergency department visit), purpose of the contact (preventive, acute), provider type (physician, nurse practitioner, trainee), all International Classification of Diseases, Ninth Revision (ICD-9) codes associated with the encounter and the active "problem list," and all prescriptions generated during the encounter. Patients were considered members of a practice group when having at least 2 encounters (office or telephone) at a practice site in the calendar year.

\section{Cohort Assembly}

All primary care office encounters from January 1 through December 31,2009, by children 18 years or younger were included in the initial cohort. The following encounters were excluded: (1) preventive encounters, to focus on "acute visits" at which the vast majority of antibiotics were prescribed; (2) visits without acute pharyngitis (ICD-9 diagnostic codes in- cluding 034.0/streptococcal sore throat, 462.0/acute pharyngitis, or $463.0 /$ acute tonsillitis), to restrict the cohort to individuals diagnosed with acute pharyngitis; (3) encounters by children with complex chronic conditions, to restrict the cohort to previously healthy children; ${ }^{19}(4)$ encounters with multiple bacterial diagnosis codes, to avoid the influence of antibiotic prescribing decisions based on other diagnoses; and (5) when relevant, encounters by children with antibiotic allergies or children who received an antibiotic prescription 3 months prior to the office visit in which they were diagnosed with GAS pharyngitis (including both office- and telephonebased prescribing), to avoid the influence of antibiotic prescribing decisions based on antibiotic allergies, the treatment of recurrent disease, or the consideration of the potential for resistant pathogens created by recent drug exposure. This generated a calendar year of acute visits for acute pharyngitis by previously healthy children to a diverse group of clinicians across urban, suburban, and rural practice settings.

\section{Outcomes}

The main outcome measures included rate of diagnosis of pharyngitis, rate of testing for GAS pharyngitis, rate of diagnosis of GAS pharyngitis, and rate of prescribing of broadspectrum antibiotics for GAS pharyngitis. Diagnosis of acute pharyngitis was defined as an encounter with an acute pharyngitis code. Testing for GAS pharyngitis was defined by a laboratory order for either a rapid GAS test or a throat culture. We defined confirmed GAS pharyngitis as an encounter with (1) acute pharyngitis, (2) a positive rapid strep test or culture, (3) an antibiotic prescription, and (4) no additional diagnosis codes for which antibiotics might be indicated assigned to the visit (see Table 1 for ICD-9 codes). The rate of prescribing of broad-spectrum antibiotics for GAS was calculated using encounters that met the criteria for confirmed GAS (denominator) with an associated broad-spectrum antibiotic prescription (numerator). Broad-spectrum antibiotics were defined as azithromycin, amoxicillin-clavulanate, and cephalosporins (generations 1, 2, or 3) based on AAP prescribing guidelines for GAS pharyngitis. ${ }^{9}$ Case definitions were validated through iterative, manual chart review of fields collected electronically as well as free text fields (eg, physical exam, assessment/plan).

\section{Statistical Analysis}

Characteristics of practices were calculated using frequencies for all variables. For across-practice analyses, logistic regression models were constructed to analyze variation in diagnosis, testing, and antibiotic prescribing. These models were standardized for patient characteristics, including age category (less than $1,1-3,4-10$, or more than 10 years old), sex, insurance type (Medicaid or non-Medicaid), and race (black or nonblack). Marginal probabilities of exposure were calculated to generate rates of diagnosis, testing, and antibiotic prescribing for GAS. For within-practice analyses, rates of 
TA B LE 1. International Classification of Diseases, Ninth Revision Codes Used to Identify Non-Group A Streptococcal (GAS) Pharyngitis Diagnoses

\begin{tabular}{lc}
\hline Diagnosis & Code \\
\hline Otitis media/externa & $380.1,380.10,380.12,381.00,381.01,381.1,381.4,382,382.0,382.00,382.01$, \\
& $382.02,382.3,382.4,382.9,384.00,384.01,384.2,385.10,386.30,388.7$ \\
Acute sinusitis & $461.8,461.9,473.9,473.2,473.1,473.0,487.1$ \\
Pneumonia & $482.9,486,485,483.8,481,519.8$ \\
Urinary tract infection & $599,599.0,788.41,788.1,590.1,590.10,590.11,590.80$ \\
Skin/soft-tissue infection & $680,680.0,680.1,680.2,680.3,680.4,680.5,680.6,680.7,680.8,680.9,681$, \\
& $681.0,681.00,681.01,681.02,681.1,681.10,681.11,681.9,682,682.0,682.1$, \\
& $682.2,682.3,682.4,682.5,682.6,682.7,682.8,682.9$ \\
Lyme disease & $088.81,919.4,711.80$ \\
Acne & 706.1 \\
Chronic sinusitis & 473.9 \\
Mycoplasma infection & $041.81,483.0,711.90$ \\
Staphylococcal infection & $041.10,041.11,041.12,041.19$ \\
Bite wound & $879.8,879.9,959.9, \mathrm{E} 906.5, \mathrm{E} 906.0, \mathrm{E} 906.3,891.0,890.0,884.0,883.0,882.0$, \\
& 881.00 \\
Oropharyngeal infection (not GAS pharyngitis) & $522.5,522.4,528.3,475,478.24$ \\
Streptococcal infection (without pharyngitis) & $034.1,041,041.00,041.01,041.1,041.09,390,040.82,566,580.0,686.9,711.40$ \\
Pertussis & $033.0,033.9$ \\
Sexually transmitted infection & $079.9,079.88,079.98,614.9,616.1,616.10$ \\
Bone/joint infection & $730.20,730.21,730.22,730.23,730.24,730.25,730.26,730.27,730.28,730.29$, \\
& $711.06,711.05,711.03,711.00$ \\
Bacterial gastroenteritis & $008.5,008.43,008.00,004.9,004.3,003.9,003.1,003.0$
\end{tabular}

diagnosis of pharyngitis, testing and diagnosis for GAS pharyngitis, and antibiotic prescribing were determined for each clinician. Only clinicians who had 10 or more encounters were included. Fixed-effects models and likelihood ratio tests were conducted to analyze variation of these rates within practices.

Stata (ver. 12.0; StataCorp) was used for statistical analysis. The Children's Hospital of Philadelphia Committee for the Protection of Human Subjects approved this study.

\section{RESULTS}

\section{Study Population}

Between January 1 and December 31, 2009, there were 208,015 children who had 630,502 outpatient encounters to 222 clinicians across 25 primary care pediatric practice groups. The number of clinicians per practice group ranged from 4 to 18 providers, and the population of children served in each practice group varied from 4,286 to 17,135 patients. Of the 399,793 acute office-based encounters, 62,055 (15.5\%) received a diagnosis code for acute pharyngitis. After excluding encounters by children with complex chronic conditions $(4,612)$ and multiple diagnoses $(4,785), 52,658$ encounters remained. Of these, 12,445 cases met our study definition for confirmed GAS pharyngitis.

\section{Streptococcal Testing}

A rapid streptococcal test or bacterial culture was performed during 50,710 (96.3\%) encounters for acute pharyngitis (Fig- ure 1). Of the 49,879 rapid streptococcal tests performed, $12,079(24.2 \%)$ were positive. Of the 37,800 encounters with a negative rapid streptococcal test, 35,888 $(94.9 \%)$ received throat cultures, 3,162 (8.6\%) of which revealed GAS (bringing the total number of positive GAS tests to 15,241). When GAS testing was positive without an acute pharyngitis ICD-9 code ( $n=109$ ), the most common diagnosis codes included scarlet fever (034.1), unspecified viral infection (079.99), and acute upper respiratory infections of unspecified site (465.9).

\section{Antibiotic Prescribing}

An antibiotic was prescribed for 17,858 (33.9\%) encounters of acute pharyngitis, 12,445 of which met the study definition for GAS pharyngitis (Figure 1). After excluding encounters by children with antibiotic allergies or antibiotic use within the prior 3 months (7,976 encounters remaining for analysis), 7,321 patients $(91.8 \%)$ received a recommended antibiotic (97\% amoxicillin). The most commonly prescribed nonrecommended antibiotics included azithromycin (2\%), amoxicillin-clavulanate $(2 \%)$, first-generation cephalosporins $(2 \%)$, second-generation cephalosporins $(0.6 \%)$, and third-generation cephalosporins $(0.8 \%)$. Of the 841 encounters for acute pharyngitis without GAS testing who received an antibiotic prescription, 412 received narrow-spectrum therapy.

\section{Variability across and within Practice Groups}

After standardizing for patient age, sex, insurance type, race, and provider characteristics, the rate of diagnosis of phar- 


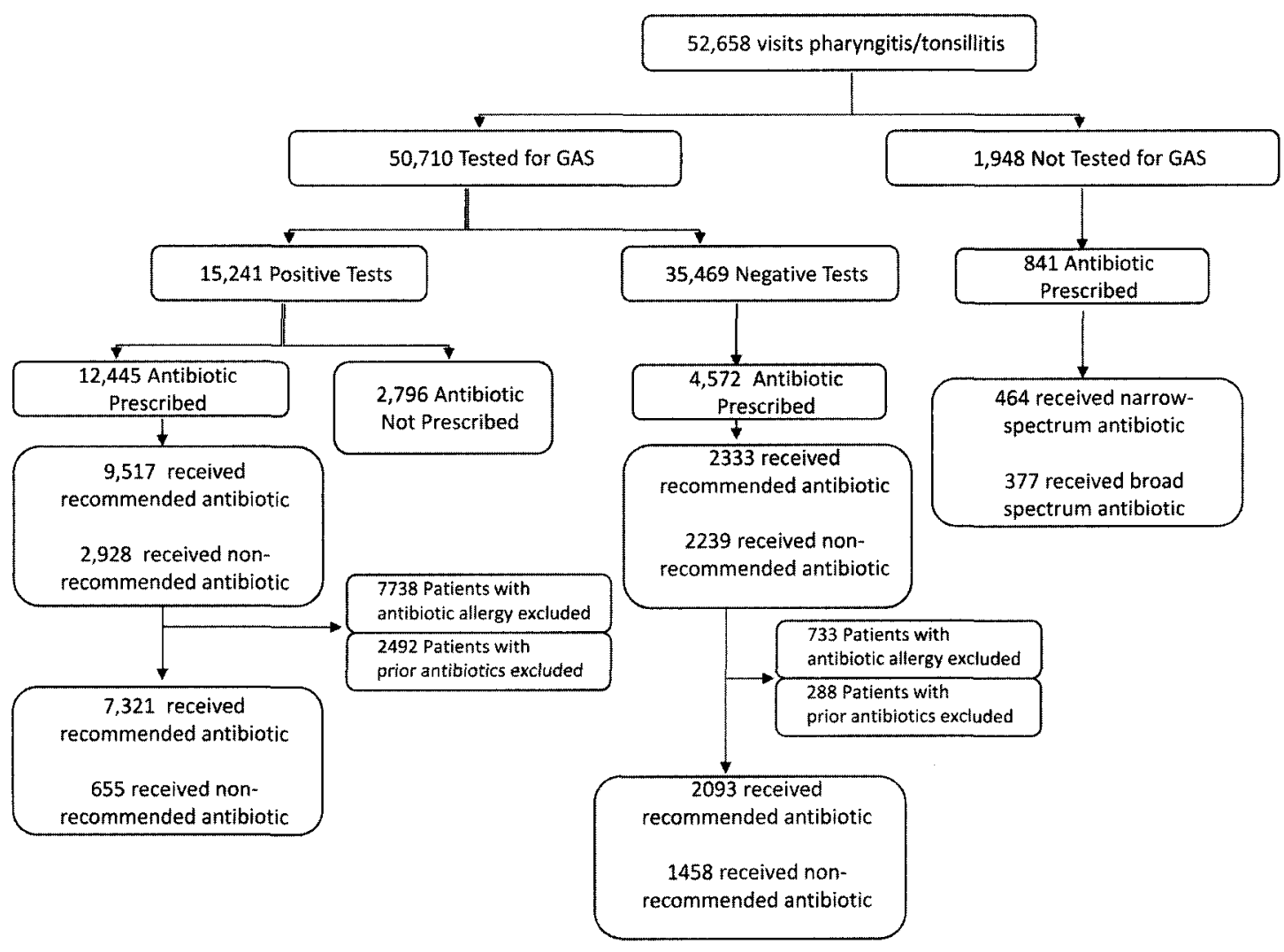

FIGURE 1. Group A streptococcal (GAS) testing and antibiotic prescribing for children presenting with acute pharyngitis.

yngitis across practice groups ranged from $10.4 \%$ to $27 \%$ of acute visits $(P<.001)$, which closely paralleled the rate of GAS testing and the rate of diagnosis of GAS pharyngitis across practice groups. Furthermore, within practice groups there was significant interclinician variability in the rate of diagnosis of pharyngitis (Figure $2 A$ ), testing for GAS (Figure $2 B$ ), and diagnosis of GAS pharyngitis (Figure $2 C ; P \leq .05$ ).

Off-guideline antibiotic prescribing for GAS pharyngitis ranged from $0 \%$ to $32.3 \%$ across practices $(P<.001)$, again excluding encounters by children with antibiotic allergies or prior antibiotic use. More than half of practices demonstrated significant within-practice variability in the rate of off-guideline antibiotic prescribing for GAS pharyngitis $(P \leq .05$; Figure $2 D$ ). Of note, 18 of the 222 clinicians accounted for half of all off-guideline antibiotic prescribing for GAS pharyngitis across the network.

\section{I S CUS S I O N}

Antibiotic overuse is one of the most important threats to public health. ${ }^{9-11,20}$ To most efficiently address this problem, interventions should prioritize high-use conditions with clear prescribing guidelines, such as acute pharyngitis. Although antibiotic overuse has been well documented, previous studies relying on national estimates have been limited by a lack of patient-specific data and an inability to compare antibiotic prescribing across clinicians and practice groups. ${ }^{6,13,17,18}$ Our study utilized a shared, comprehensive EHR of one of the nation's largest pediatric healthcare networks to evaluate differences in the diagnosis of and antibiotic use for GAS pharyngitis. We observed significant variability in rates of diagnosis, testing, and guideline-concordant treatment of GAS pharyngitis across and within primary care pediatric practices, unexplained by patient-level factors. Furthermore, a small number of clinicians accounted for a substantial proportion of the off-guideline antibiotic prescribing, suggesting that targeted interventions might effectively improve antibiotic prescribing practices for this common condition.

In this cohort, more than $15 \%$ of all acute care pediatric encounters received a diagnosis of acute pharyngitis, of which one-third received an antibiotic prescription. Although higher than the expected prevalence of GAS pharyngitis (the only indication for antibiotics among those with acute pharyngitis) ${ }^{21-24}$ the observed prescribing rate was lower than reported in prior studies, suggesting that antibiotic prescribing for acute pharyngitis might be improving. ${ }^{14,15,25}$ However, nearly half of patients who were not tested for GAS and 13\% of patients with negative GAS tests were prescribed antibiotics. These practices are at odds with AAP, CDC, and IDSA guidelines that recommend confirmatory GAS testing to dictate antibiotic therapy. ${ }^{9-11,26,27}$ 
A.

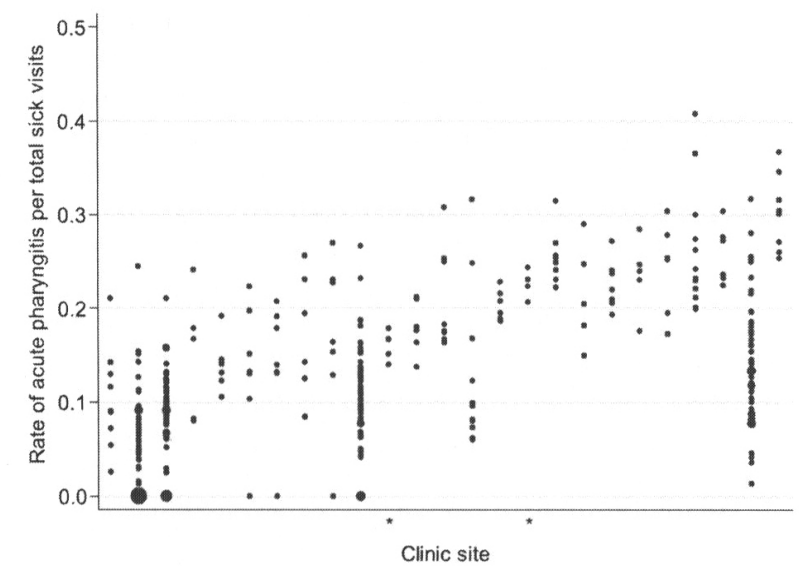

C.

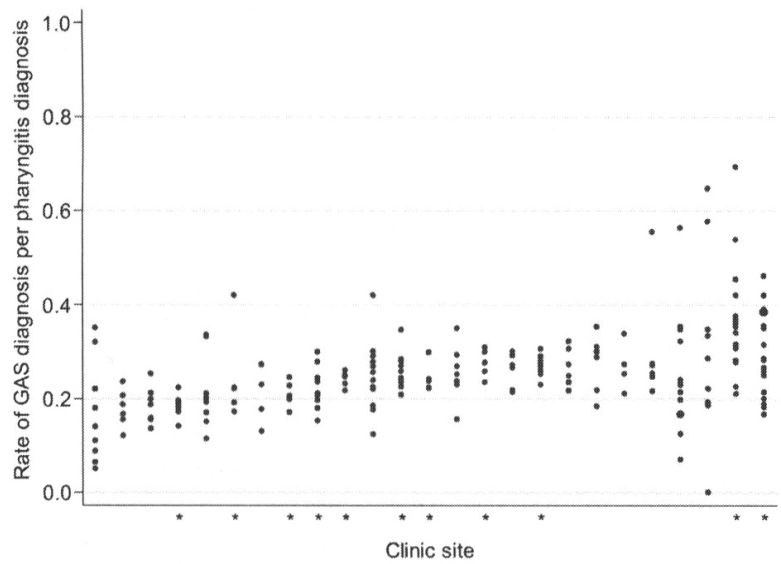

B.

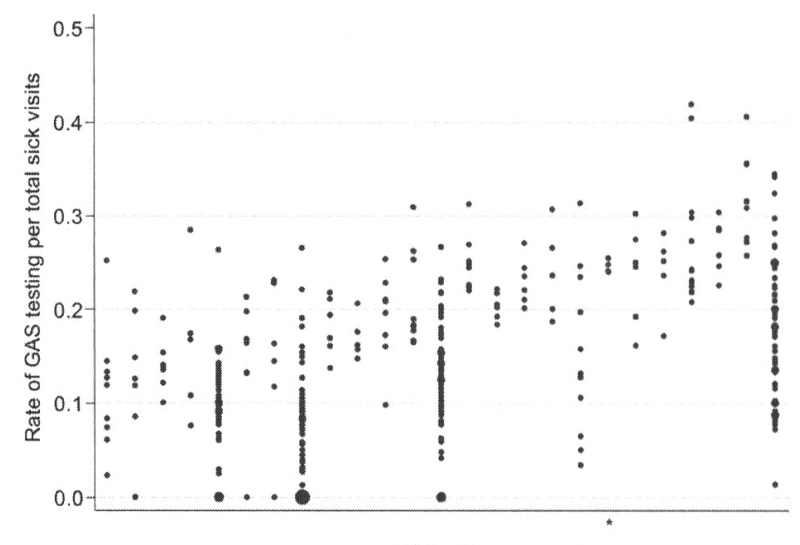

D.

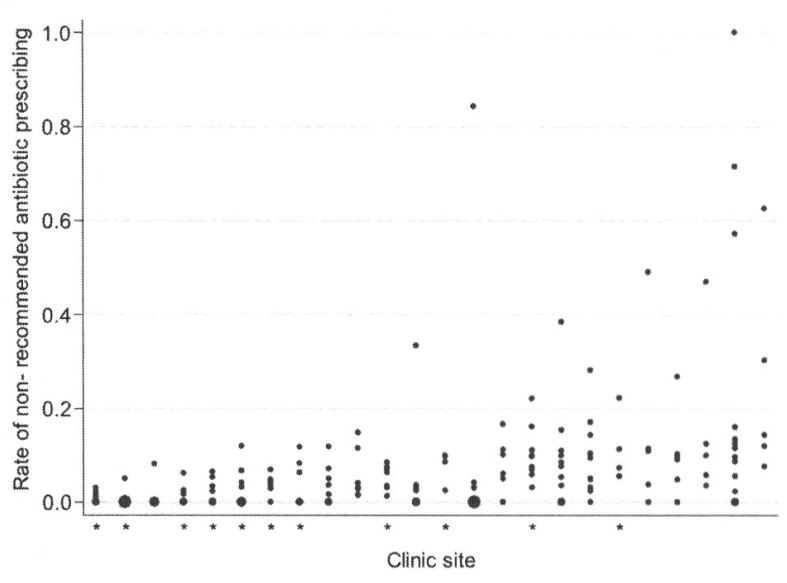

FIGURE 2. Distribution of rates of diagnosis, testing, and prescribing by practice and clinician. $A$, Rate of acute pharyngitis diagnosis within practice groups. $B$, Rate of group A streptococcal (GAS) testing within practice groups. $C$, Rate of GAS diagnosis for children with pharyngitis within practice groups. $D$, Nonrecommended antibiotic prescribing for children with GAS pharyngitis within practice groups. Individual clinicians are depicted by dots, which are arranged vertically by practice. Wider distributions reflect more variability across clinicians within the site. When multiple clinicians share a single rate, dot sizes are proportional to the number of clinicians represented. For clinic 1 in $A$ and $B$, one clinician's data produced a rate of 1.0 , a finding that suggested an error in the physician identifier; therefore, this observation was excluded (and the results for clinic 1 might understate true variability). Statistical significance of rates of clinicians within each site follows from likelihood ratio tests for the null hypothesis that those rates were the same within that site. Only sites marked with an asterisk lacked statistical significance for variability (on the basis of likelihood ratio tests for the null hypothesis that rates were the same within that site) at conventional levels $(P \leq .05)$. Only clinicians with more than or equal to 10 relevant encounters were included.

Our study also identified significant variability in the diagnosis, testing, and prescribing of broad-spectrum (offguideline) antibiotics for GAS pharyngitis both across and within primary care pediatric practices. The nearly 3-fold differences in testing for and diagnosis of GAS pharyngitis across practice groups were unexpected. This variability is unlikely to be driven by patient-level factors, given that (1) the comparisons occurred during the same calendar year; (2) analyses were standardized by patient age, sex, race, and insurance type; and (3) children with complex medical conditions, prior antibiotic use, and antibiotic allergies were excluded. Furthermore, practitioners within the same practice tested for and diagnosed GAS pharyngitis at markedly dif- ferent rates. Our clinician-level analyses revealed that practice behavior is not uniform, and, in some cases, a small proportion of clinicians accounted for much of the variability. For example, only 18 of the network's 222 clinicians wrote $50 \%$ of all broad-spectrum antibiotic prescriptions for children with GAS pharyngitis. These data support cliniciantargeted interventions to improve adherence to prescribing guidelines.

Practice guidelines recommend penicillin or amoxicillin for the treatment of GAS pharyngitis; broad-spectrum antibiotics, such as macrolides or first-generation cephalosporins, should be reserved for patients allergic to penicillins. ${ }^{9-11}$ Our analysis excluded individuals with conditions that might in- 
fluence practitioners to prescribe a broad-spectrum antibiotic, including allergies, complex chronic conditions, antibiotic use 3 months before GAS diagnosis, and additional bacterial diagnoses. Therefore, it would be expected that broad-spectrum antibiotic prescribing for GAS pharyngitis for this cohort would be close to $0 \%$. Overall, however, $8 \%$ of patients were prescribed broad-spectrum antibiotics, and the rate was as high as 30\% in some practices. Given the emergence of group A $\beta$-hemolytic streptococci resistance to macrolides (which has never been documented for penicillins), use of off-guideline therapy for GAS pharyngitis risks both exposure to unnecessary broad-spectrum antibiotics and the potential for treatment failure. , $28^{28}$

Our analysis has limitations. First, data were extracted from an EHR using ICD-9 codes to identify GAS pharyngitis. To help rectify potential misclassification, the case definition for GAS required an antibiotic prescription, a positive GAS test, an acute pharyngitis code, and the absence of an alternate bacterial infection code, which likely improved the specificity of our cohort over previous studies using only billing codes. ${ }^{13,14}$ Second, analyses were conducted using data from a regional healthcare network, which might limit the generalizability of our results. However, this network represents more than 200 clinicians serving patients from urban, suburban, and rural locations and diverse socioeconomic and racial backgrounds. Third, we were unable to distinguish between conventional and provisional ("watch and wait") antibiotic prescriptions. This practice, however, is not recommended by the AAP for this condition and, when used, should not differ substantially across clinicians and practice groups in a standardized patient population.

In conclusion, substantial differences in the diagnosis, testing, and treatment of GAS pharyngitis exist across and within primary care pediatric practices. This variability provides benchmarks for the management of a common pediatric condition that should help inform interventions to promote the judicious use of antibiotics for one of the most frequent indications for antibiotic prescribing to children.

\section{ACKNOWLEDGMENTS}

We thank Lihai Song from the Healthcare Analytics Unit of the Children's Hospital of Philadelphia's Center for Pediatric Clinical Effectiveness for preparing the data for analysis.

Financial support. This research was supported by the US Agency for Healthcare Research and Quality (contract HHSA2900200710013).

Potential conflicts of interest. All authors report no conflicts of interest relevant to this article. All authors submitted the ICMJE Form for Disclosure of Potential Conflicts of Interest, and the conflicts that the editors consider relevant to this article are disclosed here.

Address correspondence to Jeffrey S. Gerber, MD, PhD, Division of Infectious Diseases, Children's Hospital of Philadelphia, 3535 Market Street, Suite 1518, Philadelphia, PA 19104 (gerberj@email.chop.edu).

\section{REFERENCES}

1. Cosgrove SE. The relationship between antimicrobial resistance and patient outcomes: mortality, length of hospital stay, and health care costs. Clin Infect Dis 2006;42(suppl 2):S82-S89.

2. Martin JM, Green M, Barbadora KA, Wald ER. Erythromycinresistant group A streptococci in schoolchildren in Pittsburgh. $N$ Engl J Med 2002;346(16):1200-1206.

3. Tenover FC, Hughes JM. The challenges of emerging infectious diseases: development and spread of multiply-resistant bacterial pathogens. JAMA 1996;275(4):300-304.

4. Ranji SR, Steinman MA, Shojania KG, et al. Closing the Quality Gap: A Critical Analysis of Quality Improvement Strategies (Vol. 4: Antibiotic Prescribing Behavior). Report 04(06)-0051-42006. Rockville, MD: Agency for Healthcare Research and Quality, 2006.

5. Bisno AL. Acute pharyngitis. N Engl J Med 2001;344(3):205211.

6. McCaig LF, Hughes JM. Trends in antimicrobial drug prescribing among office-based physicians in the United States. JAMA 1995; 273(3):214-219.

7. Bisno AL. Acute pharyngitis: etiology and diagnosis. Pediatrics 1996;97(6 pt 2):949-954.

8. McIsaac WJ, Kellner JD, Aufricht P, Vanjaka A, Low DE. Empirical validation of guidelines for the management of pharyngitis in children and adults. JAMA 2004;291(13):1587-1595.

9. Committee on Infectious Diseases, American Academy of Pediatrics. Group A Streptococcal Infections. The Red Book. Elk Groove Village, IL: American Academy of Pediatrics, 2012.

10. Snow V, Mottur-Pilson C, Cooper RJ, Hoffman JR. Principles of appropriate antibiotic use for acute pharyngitis in adults. $A n n$ Intern Med 2001;134(6):506-508.

11. Shulman ST, Bisno AL, Clegg HW, et al. Clinical practice guideline for the diagnosis and management of group A streptococcal pharyngitis: 2012 update by the Infectious Diseases Society of America. Clin Infect Dis 2012;55(10):1279-1282.

12. Office-related antibiotic prescribing for persons aged $\leq 14$ years-United States, 1993-1994 to 2007-2008. MMWR Morb Mortal Wkly Rep 2011;60(34):1153-1156.

13. McCaig LF, Besser RE, Hughes JM. Trends in antimicrobial prescribing rates for children and adolescents. JAMA 2002;287(23): 3096-3102.

14. Gonzales R, Malone DC, Maselli JH, Sande MA. Excessive antibiotic use for acute respiratory infections in the United States. Clin Infect Dis 2001;33(6):757-762.

15. Linder JA, Bates DW, Lee GM, Finkelstein JA. Antibiotic treatment of children with sore throat. JAMA 2005;294(18):23152322.

16. Harrison PF, Lederberg J, ed. Antimicrobial Resistance: Issues and Options; Workshop Report. Washington, DC: National Academies Press, 1998.

17. Spellberg B, Guidos R, Gilbert D, et al. The epidemic of antibiotic-resistant infections: a call to action for the medical community from the Infectious Diseases Society of America. Clin Infect Dis 2008;46(2):155-164.

18. Watson RL, Dowell SF, Jayaraman M, Keyserling H, Kolczak M, Schwartz B. Antimicrobial use for pediatric upper respiratory infections: reported practice, actual practice, and parent beliefs. Pediatrics 1999;104(6):1251-1257.

19. Feudtner C, Christakis DA, Connell FA. Pediatric deaths attrib- 
utable to complex chronic conditions: a population-based study of Washington State, 1980-1997. Pediatrics 2000;106(1 pt 2): 205-209.

20. Centers for Disease Control and Prevention (CDC), US Department of Health and Human Services. Antibiotic Resistance Threats in the United States, 2013. Atlanta: CDC, 2013.

21. Walsh BT, Bookheim WW, Johnson RC, Tompkins RK. Recognition of streptococcal pharyngitis in adults. Arch Intern Med 1975;135(11):1493-1497.

22. McIsaac WJ, Goel V, To T, Low DE. The validity of a sore throat score in family practice. CMAJ 2000;163(7):811-815.

23. Centor RM, Witherspoon JM, Dalton HP, Brody CE, Link K. The diagnosis of strep throat in adults in the emergency room. Med Decis Making 1981;1(3):239-246.

24. Komaroff AL, Pass TM, Aronson MD, et al. The prediction of streptococcal pharyngitis in adults. J Gen Intern Med 1986;1(1): $1-7$.
25. Hersh AL, Shapiro DJ, Pavia AT, Shah SS. Antibiotic prescribing in ambulatory pediatrics in the United States. Pediatrics 2011; 128(6):1053-1061.

26. Appropriate testing for children with pharyngitis: percentage of children 2 to 18 years of age who were diagnosed with pharyngitis, dispensed an antibiotic, and received a group A streptococcus (strep) test for the episode. Rockville, MD: National Quality Measures Clearinghouse, Agency for Healthcare Research and Quality. http://www.qualitymeasures.ahrq.gov/content.aspx?id $=47165$. Accessed August 22, 2014.

27. Hersh AL, Jackson MA, Hicks LA. Principles of judicious antibiotic prescribing for bacterial upper respiratory tract infections in pediatrics. Pediatrics 2013;132(6):1146-1154.

28. Logan LK, McAuley JB, Shulman ST. Macrolide treatment failure in streptococcal pharyngitis resulting in acute rheumatic fever. Pediatrics 2012;129(3):e798-e802. 